\section{e0475 IMPACT OF ANAEMIA ON DEVELOPMENT OF CONTRASTINDUCED ACUTE KIDNEY INJURY AKI AFTER PERCUTANEOUS CORONARY INTERVENTIONS}

doi:10.1136/hrt.2010.208967.475

Li Wenhua, Li Dongye, Xu Tongda, Xia Yong. Department of Cardiology, Affiliated Hospital of Ouzo Medical College, 221002 Xuzhou

Objective The aim of the present study was to assess the influence of anaemia on development of contrast-induced acute kidney injury (AKI) after percutaneous coronary intervention.

Methods The subject group consisted of 1026 patients who had undergone coronary intervention procedure between January 1, 2008 and October 31, 2009. A nonionic, low osmolality contrast agent was used in our laboratory at this time. Serum creatinine values were measured before and within $48 \mathrm{~h}$ of administration of contrast agents. Contrast-induced nephropathy was defined as an increase of $30.5 \mathrm{mg} / \mathrm{dl}$ or $325 \%$ in serum creatinine concentration over baseline within $48 \mathrm{~h}$ of angiography, Anaemia as haemoglobin $(\mathrm{Hgb}) 120 \mathrm{~g} / \mathrm{l}$ in women and $130 \mathrm{~g} / \mathrm{l}$ in men.

Results Among the 1026 patients studied, 32 (3.1\%) experienced AKI after procedure. AKI occurred in $6.3 \%$ of the anaemic patients and $2.2 \%$ of the non-anaemic patients ( $p$ 0.05). Multivariate logistic regression analysis found that baseline creatinine clearance and baseline haemoglobin were independent predictors of AKI. When presence of anaemia was introduced into the multivariate model instead of baseline haemoglobin, it was also showed a significant association with AKI. Conclusion Anemia increases the incidence of AKI in patients with moderate renal dysfunction. Patients with both preexisting renal insufficiency and anaemia are at high risk of AKI. Baseline creatinine clearance and baseline haemoglobin (or anaemia) are independent predictors of AKI.

\section{Q0476 OBSERVATION THE CHANGE OF NEUTROPHIL GELATINASE- ASSOCIATED LIPOCALIN LEVELS IN PATIENTS UNDERGOING CORONARY ANGIOGRAPHY AND PERCUTANEOUS CORONARY INTERVENTION}

doi:10.1136/hrt.2010.208967.476

Li Wenhua, Han Fei, Li Dongye, Xu Tongda, Zhang Yanbin. Department of Cardiology, Affiliated Hospital of Xuzhou Medical College, JiangSu, China

Objective To observe the level of neutrophil gelatinase-associated lipocalin (NGAL) in patients undergoing coronary angiography (CAG) and /or percutaneous coronary intervention (PCI) procedure before and after operation and to explore NGAL value in predicting of contrast- induced acute renal injury (CI-AKI).

Methods 60 patients undergoing CAG and /or PCI were enrolled in this study, Serum creatinine (Scr) and urinary NGAL were detected at different time points before and after operation.

Results Of all 60 patients, the change of the Scr level were not statistically significant $(p>0.05)$, there were no case of CI-AKI. The level of urine NGAL at $6,12,24 \mathrm{~h}$ after operation was significantly increased compared with baseline $(p<0.05)$.

Conclusion Urinary NGAL has been significantly increased after coronary interventional therapy without the change of Scr levels. Urinary NGAL might be an early biomarker of prediction CI-AKI better than Scr.

\section{e0477 \\ OBESITY IS ASSOCIATED WITH INCREASED NONTARGET LESION REVASCULARIZATION AFTER PERCUTANEOUS CORONARY INTERVENTION WITH DRUGELUTING STENTS}

doi:10.1136/hrt.2010.208967.477

Wang Zhijian, Zhou Yujie, Zhao Yingxin, Shi Dongmei, Liu Yuyang, Gao Fei. Anzhen Hospital

Background Obesity is a major risk factor for developing coronary artery disease. It is less clear whether obesity accelerates progression of disease among those with established coronary disease.
Objective To evaluate the effect of obesity on repeat revascularization for target and nontarget lesions in patients undergoing percutaneous coronary intervention (PCI) with drug-eluting stents (DES) Methods We studied 4,972 patients between January 2004 and December 2006. Patients were divided into three groups according to body mass index (BMI): normal (BMI $\left.30 \mathrm{~kg} / \mathrm{m}^{2}, \mathrm{n}=1,213\right)$. Median follow-up was 26 (IR 20-33) months.

Results There was no significant difference in the cumulative incidence of target lesion revascularisation (TLR) among normalweight, overweight and obese patients (6.8\% vs $5.4 \%$ vs $6.3 \%$; $\mathrm{p}=0.186$ ). In contrast, the incidence of nontarget lesion revascularisation (nonTLR) was significantly higher in obese patients compared with normal-weight and overweight populations (8.6\% vs $5.9 \%$ vs $5.7 \% ; \mathrm{p}=0.001)$. Multivariate analysis showed that obesity was independently associated with a higher risk of nonTLR in patients without statins treatment (HR 1.53; 95\% CI 1.03 to 2.28; $\mathrm{p}=0.037)$, but not in patients with concomitant statins treatment (HR 1.29; 95\% CI 0.82 to 2.03 ; $p=0.263$ ).

Conclusions Among patients undergoing PCI with DES, obesity was not associated with TLR, but was associated with a higher risk of nonTLR. Statins use may attenuate this adverse effect of obesity on atherosclerotic progression.

\section{e0478 PIOGLITAZONE ATTENUATES PROINFLAMMATORY STATE IN NONDIABETIC PATIENTS AFTER CORONARY DRUGELUTING STENT IMPLANTATION}

doi:10.1136/hrt.2010.208967.478

Wu Yue, Zhang Weiping, Liu Weimin, Zhuo Xiaozhen, Wang Lijun, Yuan Zuyi.

Objectives The inflammatory response after DES placement has recently emerged as a major concern. We investigated the proinflammatory state on circulating peripheral blood mononuclear cells (MNC) after coronary polymer-based drug-eluting stent (DES) implantation in nondiabetic patients, and the benefit of pioglitazone (PIO), a peroxisome proliferator-activated receptor-g (PPAR-g) activator.

Methods Twenty-eight non-diabetic patients with acute coronary syndrome were randomly assigned to pioglitazone $(n=14)$ or placebo $(n=14)$ treatment. From the day after DES implantation, study medication $(30 \mathrm{mg} / \mathrm{d}$ ) for 12 weeks was given in addition to the optimal standard drug treatment, the proinflammatory state on MNC was measured at baseline and after 12 weeks.

Results PIO treatment statistically decreased plasma concentrations of interleukin-6 (IL-6), tumour necrosis factor- $\alpha$ (TNF- $\alpha$ ), migration inhibitor factor (MIF), and matrix metalloproteinase-9 (MMP-9) compared with placebo ( $\mathrm{p}=0.011,0.008,0.002$ and 0.012 , respectively), but standard therapy failed to restrain these factors. The mRNA expressions of IL- 6 , TNF- $\alpha$, MIF and MMP-9 in circulating MNC decreased significantly in the PIO group ( $p=0.031,0.012$, 0.007 and 0.022 , respectively). Interestingly, PIO prevented the loss of PPAR- $\gamma$ expression in MNC after DES implantation $(p=0.039)$. In addition, treatment with PIO enhanced the cytoplasmic IKB- $\beta$ expression ( $p=0.003$ ) and decreased the expression of the NF-kB p50 subunit and $I \kappa B-\alpha(p=0.001$ and 0.029 , respectively). Concurrently, the NF-kB DNA binding activity in MNC was significantly inhibited by PIO treatment $(p=0.004)$.

Conclusion The inflammatory responses did not reverse after implantation of DES despite standard drug therapy, whereas PIO treatment attenuated the proinflammatory state in circulating MNC. Our results suggest that PIO treatment sequentially acts through PPAR-g activation, IkB- $\beta$ induction, blockade of NF-kB activation, and inhibition of inflammatory cytokine expression. These findings demonstrate that PIO may have a novel direct protective role to modulate the local and circulating proinflammatory responses after coronary DES implantation. 